\title{
Spontaneous recovery varies inversely with the training-extinction interval
}

\author{
ROBERT A. RESCORLA \\ University of Pennsylvania, Philadelphia, Pennsylvania
}

\begin{abstract}
Four experiments found the magnitude of spontaneous recovery after extinction to be greater with a shorter interval between initial conditioning and extinction. Experiments 1 and 2 used a Pavlovian magazine approach procedure with rat subjects, Experiment 3 used an instrumental training procedure with rats, and Experiment 4 used a sign-tracking procedure with pigeons. These results are not anticipated by many accounts of spontaneous recovery that attribute it to the fading of learning that occurred during extinction.
\end{abstract}

Spontaneous recovery is one of the most widely known phenomena of associative learning. For instance, in Pavlovian conditioning, the arranging of a positive relation between a signaling conditioned stimulus (CS) and a consequent unconditioned stimulus (US) typically results in the establishment of a response to that CS. Moreover, when that relation is subsequently removed in extinction, the response deteriorates. However, introducing a period of time following that extinction routinely results from the partial restoration of responding, so-called spontaneous recovery (e.g., Pavlov, 1927). A similar pattern results from instrumental learning when a response is first paired with a reinforcer and then the reinforcer is omitted (e.g., Ellson, 1938).

Results such as these are commonly interpreted in an associative framework, according to which pairings of a CS and a US result in the development of an association between the internal representations of the two events. The observation of spontaneous recovery after the extinction is usually interpreted as meaning that extinction did not fully remove the original CS-US association (e.g., Bouton, 1991; Mackintosh, 1974). Instead, it is frequently argued that extinction produced response decrement because it resulted in some new learning that counteracted the original learning, while leaving the original learning at least partially in place. Spontaneous recovery is envisioned as resulting because the counteracting learning that developed in extinction diminishes in its impact with the passage of time.

Although accounts of why the impact of extinction diminishes vary widely, one common intuition is that when the organism has had two conflicting experiences, the relatively more recent experience will have a relatively

This research was supported by National Institutes of Health Grant R01 MH 67848. Correspondence concerning this article should be addressed to R. A. Rescorla, Department of Psychology, University of Pennsylvania, 3815 Walnut Street, Philadelphia, PA 19104 (e-mail: rescorla@cattell.psych.upenn.edu). greater effect on performance. Immediately after extinction, the experience of nonreinforcement is quite recent relative to the prior experience of reinforcement; however, with the passage of time, the two experiences become increasingly more similar in recency, with the result that the original learning contributes relatively more to performance. Hence, the behavior partially returns, generating spontaneous recovery.

This intuition suggests that spontaneous recovery might be influenced not only by the extinction-test interval but also by the acquisition-extinction interval. If there is a longer interval between acquisition and extinction, then the recency advantage of the extinction experience could remain more substantial at the time of the test for spontaneous recovery. Hence, the contribution of original training would continue to be diminished, and spontaneous recovery would be relatively reduced. That is, with a fixed time interval between extinction and test, one might expect less spontaneous recovery the greater the time interval between original learning and extinction.

One theoretical framework that captures this intuition more explicitly is the temporal weighting rule model proposed by Devenport (Devenport, 1998; Devenport, Hill, Wilson, \& Ogden, 1997). According to that model, when an animal has multiple experiences with a stimulus prior to a test, it weights those experiences by the relative time that has passed between each one and the test. The result is that more recent experiences are weighted more heavily but that the differential weighting diminishes as time passes. Although Devenport (1998) explicitly noted the implication that spontaneous recovery should be both a positive function of the extinction-test interval and a negative function of the acquisition-extinction interval, there are few data to evaluate the latter implication.

There is some evidence from interference paradigms other than acquisition and extinction that the interval between two contrary learning experiences affects subsequent recall. For instance, Underwood and Freund (1968) varied the interval between the learning of two lists in a human study of proactive inhibition. They found that the 
shorter the interlist interval, the greater the negative impact of the first learned list on the delayed recall of items from the second learned list. Gordon and Spear (1973) found a similar result when they preceded active avoidance training with competitive passive avoidance training and tested after a 1-h delay. The shorter the delay between the two experiences, the greater the interference of the first learning with responding established in the second learning. However, they did not observe the same pattern with a $24-\mathrm{h}$ delay before testing. One may view these procedures as analogous to an acquisition and extinction procedure that also involves sequential exposure to two competing learning experiences. The analogous result would be the greater reemergence of the behavior learned in original acquisition as the interval between acquisition and extinction becomes shorter.

The goal of the present experiments was to provide some initial information on the importance of the trainingextinction interval for the observation of spontaneous recovery after extinction. Experiments 1 and 2 used a popular contemporary Pavlovian conditioning preparation, the magazine approach, with rat subjects. In this preparation, a diffuse auditory or visual stimulus signals the arrival of food in the magazine. As conditioning develops, the animal increasingly approaches and enters the food magazine during the signal. Although this paradigm surely contains an instrumental component, it is widely interpreted in terms of Pavlovian conditioning. Experiment 3 used an instrumental training procedure with rats in which a leverpress or a chainpull produced a food reinforcement. Experiment 4 used a sign-tracking procedure with pigeon subjects in which a localized visual stimulus signaled the coming of grain. As conditioning proceeds in this prepa- ration, the animal increasingly directs pecking at the signal. In each case, the original training was followed by extinction and then by a test of spontaneous recovery. In each case, stimuli or responses differed only in the time interval between original learning and extinction.

\section{EXPERIMENT 1}

This experiment was an initial investigation of the importance of the training-extinction interval using a Pavlovian magazine approach conditioning procedure in rats. Each animal first received repeated pairings of one stimulus (either a noise or a light) with food. Then it received repeated pairings of a second stimulus (either a light or a noise) with food. Then both stimuli received a single common extinction session and, after a 48-h interval, a common test for spontaneous recovery from that extinction. The expectation was that the magazine approach behavior would be depressed by extinction but would recover with time. The question of interest was whether that recovery would be differential for the two stimuli that had been trained with different trainingextinction intervals.

\section{Method}

\section{Subjects and Apparatus}

The subjects were 16 male Sprague Dawley rats about 90 days old. They were housed in individual cages and placed on a food deprivation regime that kept them at $80 \%$ of their ad-lib body weight for the course of the experiment. They had free access to water in the home cage.

The apparatus consisted of eight operant chambers measuring $22.9 \times 20.3 \times 20.3 \mathrm{~cm}$, identical to those used in previous reports (e.g., Colwill \& Rescorla, 1985). The two end walls of each cham-

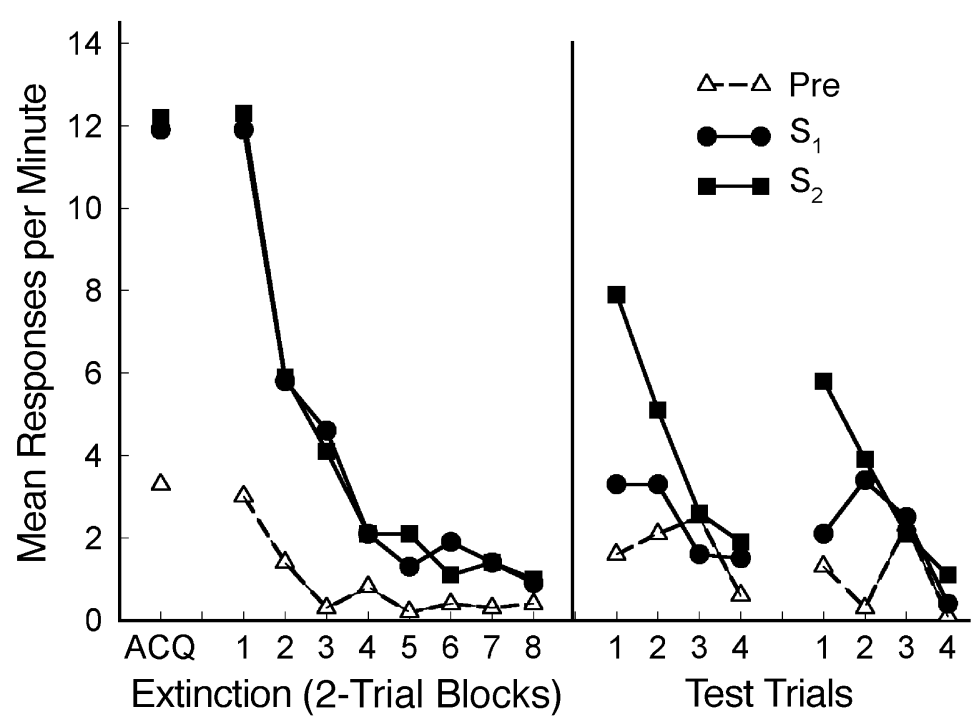

Figure 1. Extinction and test trials of Experiment 1. Responding is shown at the end of acquisition, in extinction, and in test during the prestimulus (pre) period as well as during stimuli for which training had been administered distantly from (S1) or proximally to (S2) extinction. 
ber were aluminum; the side walls and ceiling were clear Plexiglas. The floor of the chamber was composed of $0.48-\mathrm{cm}$ stainless steel rods, spaced $1.9 \mathrm{~cm}$ apart, center to center. Each chamber had a recessed food magazine located behind a $4-\mathrm{cm} \times 4-\mathrm{cm}$ opening in the center of one end wall. An infrared detector and emitter system was mounted on the side walls of the magazine, $1.5 \mathrm{~cm}$ behind the opening and $1.5 \mathrm{~cm}$ from the floor, permitting automatic recording of head movements into the magazine.

Each chamber was enclosed in a sound- and light-resistant shell. Mounted on the inside wall of this shell were two speakers, one of which permitted the presentation of a white noise $(\mathrm{N})$ measuring approximately $76 \mathrm{~dB}$ re $20 \mu \mathrm{N} / \mathrm{m}^{2}$ against a background level of $62 \mathrm{~dB}$. Also mounted on that wall was a $6-\mathrm{W}$ bulb that could be illuminated to provide a light (L) stimulus during the otherwise dark session. A food dispenser containing 45-mg pellets (P. J. Noyes Co., Formula A) allowed delivery of pellets into the food magazine.

Experimental events were controlled and recorded automatically by relays and microprocessors located in an adjoining room.

\section{Procedure}

Magazine training. On the first day, the animals received a 20min magazine training session, during which 20 noncontingent deliveries of pellets were given, at time intervals variable around a mean of $1 \mathrm{~min}$.

Conditioning. On each of the next 8 days, all animals received Pavlovian conditioning to either $\mathrm{N}$ or L. Each daily session contained sixteen 30 -sec presentations of one of the two stimuli, terminating in the delivery of a food pellet. On the next 8 days, each animal received the same treatment with the alternative stimulus. The intertrial interval (ITI), defined from trial onset to trial onset, was variable around a mean of $2.5 \mathrm{~min}$ in these and all subsequent sessions. The number of photo beam interruptions by head entry into the magazine was recorded during each $30-\mathrm{sec}$ stimulus and during the 30 -sec stimulus-free period preceding each stimulus.

Extinction. On the day following the final conditioning session, each animal received a single session in which both $\mathrm{L}$ and $\mathrm{N}$ were nonreinforced. Each stimulus was presented 16 times with a mean ITI of $2.5 \mathrm{~min}$. The order of the stimulus presentations was counterbalanced, with the restriction that no more than 3 presentations in a row could be of a particular stimulus.

Tests. On the following day, the animals remained in their home cages but were maintained on their food deprivation regime. On the next day, the animals received a test session that contained four nonreinforced presentations each of $\mathrm{L}$ and $\mathrm{N}$. This session was followed by an additional 5 days of no treatment and a second test, identical to the first.

\section{Results and Discussion}

Initial conditioning proceeded without incident. On the final day of conditioning, the mean responses per minute were 11.9 and 12.2 for the first-trained and secondtrained stimuli, respectively. The standard errors of the mean (SEMs) were 2.1. There were no reliable differences as a function of stimulus identity.

The left-hand side of Figure 1 shows responding during the course of extinction for the prestimulus period and for the first- (S1) and second- (S2) trained stimuli. It is clear that there were no differences between the stimuli over the course of extinction. That observation suggests that at the point of extinction the two stimuli had similar strengths. Their mere difference in time since conditioning did not result in differential strength at the time of extinction.
The right-hand portion of Figure 1 shows responding during the test sessions given 2 and 7 days after extinction. As the figure indicates, there was recovery of responding during the beginning of each test session relative to that at the end of extinction. Of most interest, the magnitude of that recovery was greater for the secondtrained stimulus: On both test days, there was greater responding to the second-trained stimulus on the first trial of the test [Wilcoxon $T \mathrm{~s}(16)=26,27, p \mathrm{~s}<.05]$ ]. The SEMs ranged from 0.2 to 0.9 .

This result suggests that spontaneous recovery is greater for a stimulus that is trained closer in time to extinction than for one that is trained in the more distant past. That difference does not appear to be attributable to the overall conditioned strength of the stimuli, because the course of extinction was virtually identical for the two stimuli. It was only with the passage of time after extinction that the difference emerged.

\section{EXPERIMENT 2}

Although Experiment 1 suggests that more distantly trained stimuli will show less spontaneous recovery, the design of that study confounded differences in the training-extinction interval of two stimuli with differences in the order of training. To some extent, this confounding is a necessary consequence of using a withinsubjects design in which two different stimuli are trained at different temporal distances from a common extinction period. The goal of Experiment 2 was to replicate the procedures of Experiment 1, retaining the sensitivity of a within-subjects comparison, while reducing the differences in order of training. For this purpose, Experiment 2 gave most of the initial training of both stimuli in the same sessions in an intermixed fashion. Both of the stimuli then received several sessions of additional training, but for one stimulus that additional training occurred immediately, for the other it occurred after a delay. Then both stimuli were extinguished, given a rest, and tested for spontaneous recovery. This procedure ensured that both stimuli had the same initial training but that they still differed in the time between the most recent training and extinction.

\section{Method}

\section{Subjects and Apparatus}

The subjects were 16 rats of the same type and maintained in the same manner as in Experiment 1. The apparatus was that of Experiment 1.

\section{Procedure}

The procedure was very similar to that of Experiment 1, except that initial training was divided into three phases. In the first phase, the animals all received 6 days of conditioning, each containing 16 reinforced presentations of both $\mathrm{L}$ and $\mathrm{N}$. In the second phase, one of those stimuli received 2 days of additional training, each containing 16 reinforced presentations. For half of the animals, this stimulus was L, for the other half it was N. Following a 6-day rest period, in the third phase all animals received 2 days of additional 
training with the other stimulus, each training including 16 reinforced presentations of the alternative stimulus.

This last phase was followed by an extinction and test sequence identical to that of Experiment 1

\section{Results and Discussion}

Figure 2 shows the results of this experiment from the end of acquisition, over the course of extinction, and in the recovery tests. The results were very similar to those of Experiment 1. Prior to and throughout extinction, S1 and S2 showed highly similar levels of performance. However, on the initial trial of the test sessions, there was greater responding to the stimulus trained more proximally to extinction (S2). This difference was reliable on both the first $[T(16)=21.5, p<.05]$ and the second $[T(8)=2, p<.05]$ test sessions. The SEMs ranged from 1.0 to 1.2 .

These results replicate those of Experiment 1. The stimulus that had received its last training in the more distant past showed less spontaneous recovery. The fact that the stimuli received most of their training at the same time, and showed such similar performance during the course of extinction itself, suggests that the training procedures had not resulted in stimuli with overall differences in associative strength. Moreover, they suggest that it is the time of most recent training, rather that differences in order of initial conditioning, that produces this effect.

\section{EXPERIMENT 3}

Spontaneous recovery is a property of extinction not only with Pavlovian but also with instrumental learning. It is therefore of interest to ask whether the trainingextinction interval has the same effect on spontaneous recovery after extinction of instrumental learning as it does after the extinction of Pavlovian conditioning. Experiment 3 addressed this question.

The procedure was modeled on that of Experiment 2, except that instrumental responses replaced Pavlovian CSs. Initially, the rats received reinforcement of two instrumental responses, leverpress and chainpull. Then they received an additional 2 days of training with one of those responses. After a rest interval, they received 2 days of training with the other response. This final training was followed by extinction and a test for spontaneous recovery.

\section{Method}

\section{Subjects and Apparatus}

The subjects were 16 rats of the same type and maintained in the same manner as in Experiment 1. The apparatus was that of Experiment 1 , adapted so that either a lever could be made available to the left of the food magazine or a chain could be suspended from the ceiling to the right of the magazine.

\section{Procedure}

After initial magazine training identical to that of Experiment 1, all animals were trained to press the lever and to pull the chain to receive food reinforcement. Each of two training sessions allowed one response to earn approximately 25 pellets on a continuous reinforcement schedule. On each of the next 5 days, all animals received variable interval (VI) training with the lever and with the chain. Each day contained two 20-min sessions, one with each manipulandum, during which responding was reinforced on a VI 1-min schedule.

On each of the next 2 days, the animals received only a single training session, with one of the manipulanda reinforced on a VI 1min schedule. For half of the animals, the manipulandum was the lever, for the other half it was the chain. After a 6-day rest period, all animals received two similar sessions with the other manipulandum. On the following day, all animals received two 20-min extinction sessions, one with each manipulandum, during which no reinforcers were delivered. The order in which these sessions was

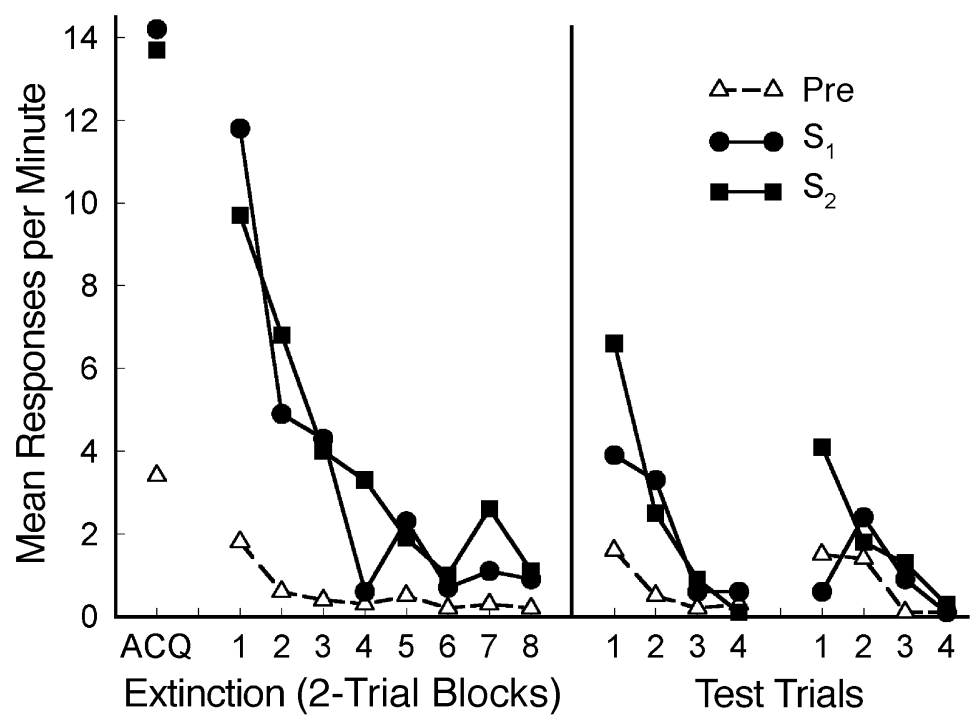

Figure 2. Extinction and test trials of Experiment 2. Responding is shown at the end of acquisition, in extinction, and in test during the prestimulus (pre) period as well as during stimuli for which training had been administered distantly from (S1) or proximally to (S2) extinction. 
administered was balanced with regard to the identity of the manipulanda and their prior histories.

After a 48-h period, all animals were tested for spontaneous recovery. This test consisted of two 8-min sessions, during which one manipulandum was present but nonreinforced. The order in which these sessions were administered was again counterbalanced with regard to identity of the manipulandum and the history of training and extinction. Following an additional 5-day rest period, a second test session, identical to the first, was administered.

\section{Results and Discussion}

Initial response training proceeded without incident. At no stage of the experiment was there a reliable difference between responding to the lever and the chain; consequently, the data are presented for those responses combined.

Figure 3 shows responding at the end of training, over the course of extinction, and during the tests. Responses on the first-trained (R1) and second-trained (R2) devices are clearly highly similar in acquisition and during extinction. Moreover, responses with both manipulanda show signs of spontaneous recovery in both test sessions. However, the magnitude of that recovery appears to be greater for R2. That difference proved reliable for the first 2-min block of each of the test sessions $[T \mathrm{~s}(16)=$ $29,24.5, p \mathrm{~s}<.05]$. The $S E M \mathrm{~s}$ ranged from 0.6 to 0.8 .

These results replicate, for the case of instrumental learning, the findings of Experiments 1 and 2, that more distant training results in reduced spontaneous recovery.

\section{EXPERIMENT 4}

Experiment 4 sought to replicate, using a different organism and conditioning preparation, the observations of
Experiment 1. Pigeons were given a Pavlovian signtracking procedure in which three different localized visual stimuli were successively paired with grain at three different times prior to extinction. Then all three stimuli were extinguished in the same session, and after a rest period, the animals were tested for spontaneous recovery.

\section{Method}

\section{Subjects and Apparatus}

The subjects were 15 female White Carneau pigeons about 1 year old, originally purchased from Palmetto Pigeon Plant. They had previously participated in another sign-tracking experiment that had used different stimuli. The assignment of birds to groups in the present experiment was random with respect to their previous treatments. They were housed in pairs and maintained at $80 \%$ of their free-feeding weights.

The apparatus was eight identical operant chambers, each measuring $27 \times 27 \times 35 \mathrm{~cm}$. The metal front panel of each chamber had a $5-\mathrm{cm} \times 5-\mathrm{cm}$ food magazine in its center, located $5 \mathrm{~cm}$ above the wire mesh floor. An 11.8-cm $\times 14.5-\mathrm{cm}$ response key, constructed of clear Lucite acrylic, was centered $9 \mathrm{~cm}$ above the magazine, behind a $10-\times 8-\mathrm{cm}$ rectangular opening in the chamber wall. Mounted behind the top edge of this response key was a set of relay contacts, the closure of which detected pecking. Located $2 \mathrm{~mm}$ behind the response key was a Magnavox (model CK3923) color television connected to a computer programmed to generate and display visual stimuli. In four of the boxes, a black opaque strip blocked the top half of the screen from view, and a strip blocked the bottom half of the screen in the other four boxes. Four visual stimuli could be presented in the middle of the visible portion of this screen: a red 5- $\times 23-\mathrm{mm}$ bar oriented $45^{\circ}$ from the vertical, a vertical blue bar of the same dimensions, a horizontal yellow bar of the same size oriented horizontally, and a circular stimulus composed of a set of black and white concentric circles, each $4 \mathrm{~mm}$ thick, with an outer diameter of $20 \mathrm{~mm}$.

The other three walls and the ceilings of the chambers were composed of clear Plexiglas. These chambers were placed in sound- and

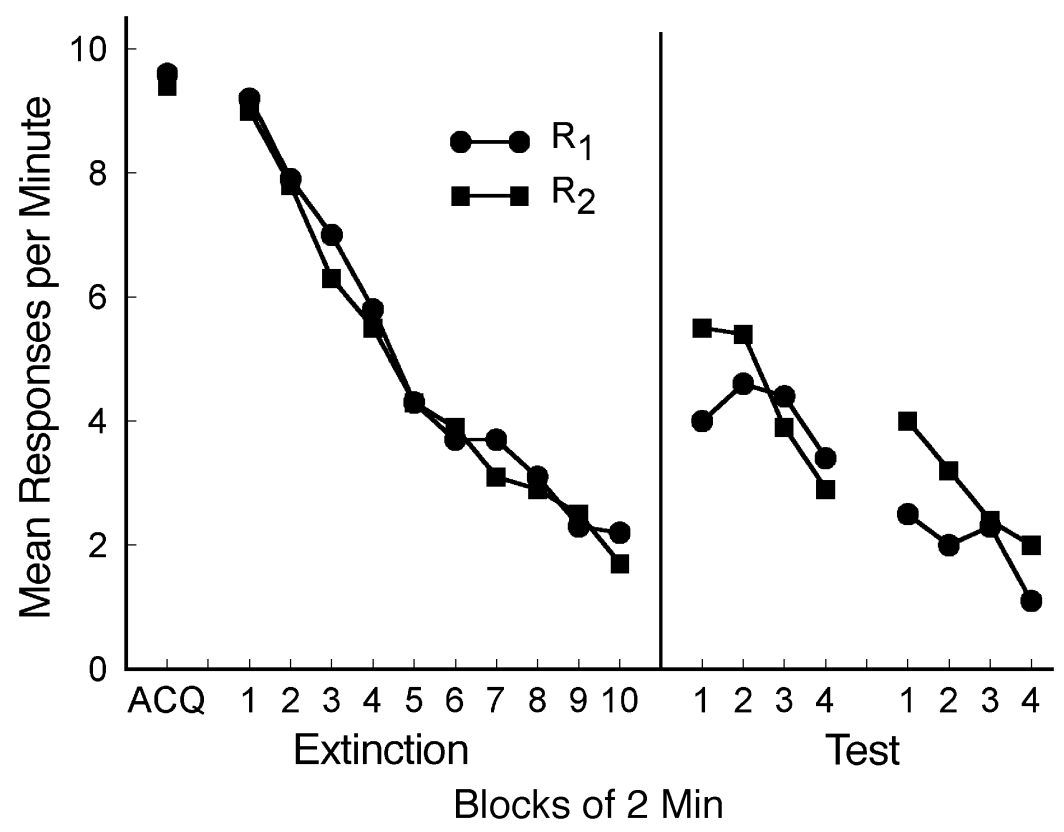

Figure 3. Extinction and test responding of Experiment 3. Responding is shown for responses that had been trained distantly from (R1) or proximally to (R2) extinction. 
light-attenuating shells with ventilation fans providing background noise of $62 \mathrm{~dB}$ re $20 \mu \mathrm{N} / \mathrm{m}^{2}$. On the rear wall of those shells was mounted a $6-\mathrm{W}$ bulb that was continuously illuminated during the session, except during the operation of the food hopper. That hopper contained Purina Pigeon grain. Experimental events were automatically controlled by relay equipment and microprocessors located in an adjoining room.

\section{Procedure}

In order to ensure initially low levels of responding to the stimuli, the experiment began with a preexposure phase. On each of 9 days, the animals were given 12 nonreinforced exposures to each of the colored bars. In addition, they received 12 reinforced presentations of the circle during this and all subsequent sessions. Each session began with 3 reinforced presentations of the circle. This procedure of beginning each session with the circle and continuing to reinforce circle presentations in the session was adopted in order to keep the birds active and to reduce the disruption that is commonly observed at the beginning of a sign-tracking session. This starting procedure is routinely used in our laboratory for this purpose.

On each of the next 5 days, the birds received reinforced presentations of one of the three colored bars. Each day contained 125 sec presentations of the circle mixed with 245 -sec presentations of one of the bars, all reinforced. Each of the colored bars was used for five birds.

After a 9-day rest, during which the animals remained in their home cages but continued on their feeding regime, each bird received a 5-day conditioning phase identical to the previous training except that a different colored bar was reinforced. This second phase was followed by a second 9-day rest and a third conditioning phase with the remaining colored bar. The identities of the second and third trained bars were counterbalanced across animals.

On each of the next 3 days, all animals received an extinction treatment identical to that used in pretesting: 12 reinforced presentations of the circle intermixed with 12 nonreinforced presentations of each of the colored bars. After a rest of 7 days, all animals were tested in a session that contained 12 reinforced presentations of the circle intermixed with 6 nonreinforced presentations of each colored bar.

\section{Results and Discussion}

Because one bird showed an unusually high rate of responding to the three colored lines during preexposure, that phase was continued for 9 days. At the end of that period, the mean responses per minute were 165.2 and 1.7 for the circle stimulus and the colored lines, respectively.

Figure 4 displays responding at the end of conditioning, over the course of the three extinction sessions, and in the final test. The three stimuli showed similar levels of responding at the end of acquisition. Responding declined over the course of extinction, with some indications of between-sessions spontaneous recovery. Although none of the differences was reliable during extinction, the between-sessions recovery appeared to be numerically greatest for the most recently trained stimulus (S3) and least for the first-trained stimulus (S1).

The right-hand side of Figure 4 shows the results of responding in the test session, plotted for blocks of two trials. Numerically, the amounts of responding during this test were greatest for S3 and least for S1. However, the only reliable differences were those between responding to $\mathrm{S} 3$ and to the other two stimuli $\left[T_{\mathrm{s}}(12)<14, p \mathrm{~s}<.05\right]$. The SEMs ranged from 7.2 to 12.6. The failure to observe a reliable difference in responding to $\mathrm{S} 1$ and $\mathrm{S} 2$ may re- flect the fact that the interval between training and extinction was so substantial for both stimuli.

Overall, these results are in agreement with those of previous experiments. The stimulus that received training nearest to the extinction treatment showed the greatest spontaneous recovery.

\section{GENERAL DISCUSSION}

The observation that spontaneous recovery is greater for stimuli trained in greater temporal proximity to extinction seems relevant to the evaluation of various accounts that have been offered for that recovery. All accounts of spontaneous recovery assume that extinction has left some of the original learning intact, with some new learning occurring during extinction, but they differ in what happens with the passage of time following extinction. One class of accounts suggests that the depressive process that develops during extinction itself diminishes with time, allowing the original learning to reappear. Another class envisions the animal as fully retaining what was learned both in original conditioning and in extinction but changing its relative use of those two with the passage of time.

The most prominent examples of the first class are theories that point to "inhibition" as something that develops during extinction but then fades with the passage of time. Many different kinds of inhibitory process have been suggested. Some authors (e.g., Pavlov, 1927; Robbins, 1990) have suggested that repeated nonreinforced presentation of the CS leads to a CS-specific inhibitory process, somewhat akin to sensory fatigue. For Pavlov, the inhibition located in the CS grows with repeated CS presentations but then fades in time. For Robbins, the processing of the CS diminishes during extinction but then recovers with time. Others (e.g., Rescorla \& Cunningham, 1978) have argued that extinction leads to a US-specific inhibition, such that stimuli previously paired with a US would be less effective. Again, that inhibition has been suggested to fade with time. Many authors have noted that nonreinforcement in other paradigms produces a kind of associative inhibition. For instance, Konorski $(1948,1967)$ and Rescorla and Wagner (1972) envisioned that nonreinforcement could engender a kind of inhibitory association that is the opposite of the excitatory association of original conditioning. There is some evidence that the power of this inhibition may diminish with time (e.g., Hendersen, 1978). Some (e.g., Hull, 1943; Rescorla, 1993) have also suggested a kind of responsebased inhibition, according to which the animal becomes less inclined to make a particular response as a result of extinction. For Hull, that inhibition is akin to response fatigue and recovers with time. For Rescorla, the response develops an inhibitory association with the stimulus, an association that decreases with time. Most recently, it has been proposed (e.g., Bouton, 1991) that the inhibition that develops in extinction might be analogous to occasion setting. According to this view, extinction leads to the tem- 


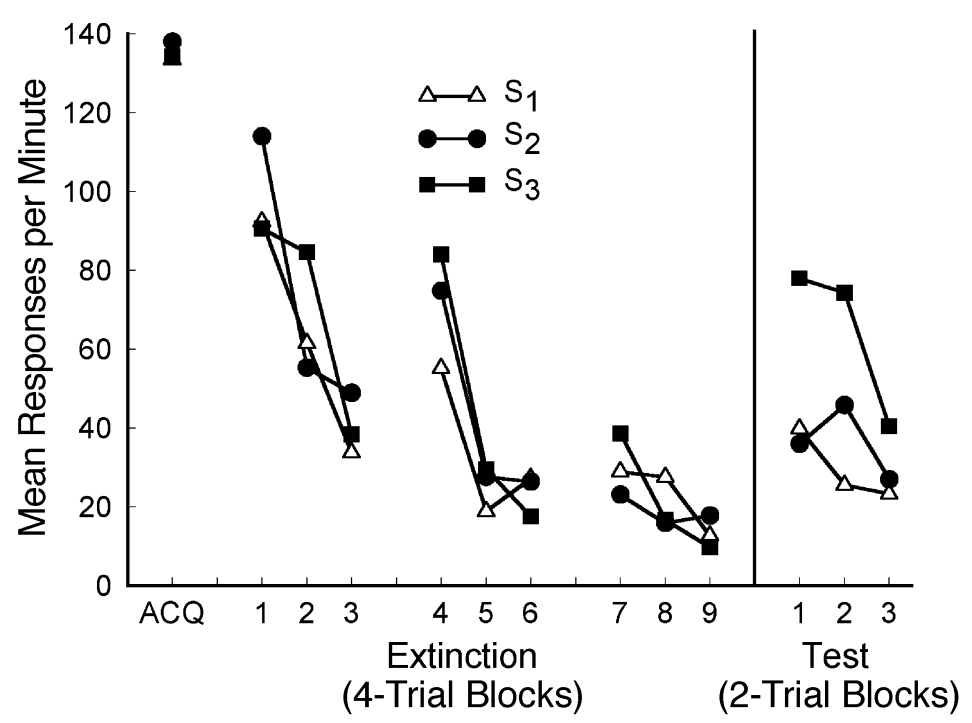

Figure 4. Extinction and test responding of Experiment 4. Responding is shown to stimuli trained distantly from (S1), more proximally to (S2), and most proximally to (S3) extinction.

poral context serving as a modulator for the new learning that occurs in extinction. As time passes, that context changes, reducing the influence of the modulation.

These inhibitory accounts differ not only in the nature of the inhibition but also in the degree to which they provide a rationale for changes with time. Some, such as Pavlov (1927), Hull (1943), and Bouton (1991), see changes over time as an inherent feature of the inhibitory process. But for others, such as Konorski (1967), Rescorla and Cunningham (1978), and Robbins (1990), no separate rationale is given for changes in the inhibitory process with time. Rather, the fading with time is an arbitrary assumption seemingly designed simply to explain spontaneous recovery.

However, in each of these cases, spontaneous recovery is seen as attributable to the change with time in the status of what was learned in extinction. Each sees the extinction learning as somehow qualitatively different from the original learning, such that it is specifically sensitive to the passage of time. Essentially, each of these theories assumes that there is little or no change over time in the original learning but a fading in time of the effectiveness of what was learned in extinction. Consequently, all of these theories suggest that spontaneous recovery should be dependent on the passage of time following extinction. In none of these theories is there reason to believe that this fading could depend on the temporal interval between training and test. As a result, without additional assumptions, none of these approaches provides a natural account of the data observed here.

Another class of accounts of spontaneous recovery makes more explicit reference to the comparison of what is learned during original acquisition and extinction. According to these accounts, what was learned during both acquisition and extinction remains fully intact, and performance is a function of their relative effectiveness. For instance, one account of spontaneous recovery is that performance depends on the relative similarity of the test session to the training and extinction sessions. A test given immediately after extinction might contain stimulus elements that are highly similar to those of extinction, but with the passage of time the relative similarity of test to extinction should diminish, allowing more use of the original learning (e.g., Spear, 1971). Although using a somewhat different language, Devenport's (1998) temporal weighting rule is similar. In that theory, too, the absolute proximity of the test to extinction is not what matters, but rather the test-extinction proximity relative to the test-training proximity. Because these theories suppose that the temporal or stimulus similarity of the test to both the extinction and the original training is important, they can provide an account of why the training-test interval might affect spontaneous recovery.

In order to vary the conditioning-extinction interval while holding constant the extinction-test interval, the present experiments necessarily confounded the interval between conditioning and test. Experiments 2 and 3 found an effect of the conditioning- extinction interval while attempting to reduce such confounding by doing the bulk of the conditioning at a common time. Together with the observation of similar responding during extinction itself, these results suggest that the simple distance from initial conditioning is not the primary determinant of performance in these experiments. However, it should be noted that elsewhere Rescorla (1997) has employed an alternative general design for the study of spontaneous recovery that contains a similar confound. In those experiments, multiple stimuli were conditioned in a session 
and tested after a common interval, but those stimuli differed in whether their extinction occurred early or late in the interval between conditioning and test. Stimuli extinguished early had an opportunity to develop spontaneous recovery after extinction, whereas those extinguished later did not. The present results suggest that this alternative design should be able to show an especially large magnitude of spontaneous recovery, but they also strengthen the recommendation that multiple designs be used for the study of spontaneous recovery (Rescorla, 1997) in order to separate out unavoidable confoundings.

Spontaneous recovery is a highly robust phenomenon, with many potential sources. The present finding that the passage of time between training and test affects the size of recovery is consistent with accounts in terms of the relative likelihood of retrieval of contradictory experiences. However, other instances of spontaneous recovery, such as that following sequential training with two equally valued outcomes (e.g., Rescorla, 1997), seem less consistent with such accounts. Consequently, it remains likely that spontaneous recovery is a multiply determined phenomenon.

\section{REFERENCES}

Bouton, M. E. (1991). Context and retrieval in extinction and in other examples of interference in simple associative learning. In L. W. Dachowski \& C. F. Flaherty (Eds.), Current topics in animal learning: Brain, emotion, and cognition (pp. 25-53). Hillsdale, NJ: Erlbaum.

Colwill, R. M., \& Rescorla, R. A. (1985). Postconditioning devaluation of a reinforcer affects instrumental responding. Journal of Experimental Psychology: Animal Behavior Processes, 11, 120-132.

DEVENPORT, L. D. (1998). Spontaneous recovery without interference: Why remembering is adaptive. Animal Learning \& Behavior, $\mathbf{2 6}_{2}$ 172-181.

Devenport, L. D., Hill, T., Wilson, M., \& Ogden, E. (1997). Tracking and averaging in a variable environment: A transition rule. Journal of Experimental Psychology: Animal Behavior Processes, 23, 450-460.
Ellson, D. G. (1938). Quantitative studies of the interaction of simple habits: I. Recovery from specific and generalized effects of extinction. Journal of Experimental Psychology, 23, 339-358.

GoRdon, W. C., \& SpeAR, N. E. (1973). Effect of reactivation of a previously acquired memory on the interaction between memories in the rat. Journal of Experimental Psychology, 99, 349-355.

Hendersen, R. W. (1978). Forgetting of conditioned fear inhibition. Learning \& Motivation, 9, 16-30.

Hull, C. L. (1943). Principles of behavior. New York: AppletonCentury.

Konorski, J. (1948). Conditioned reflexes and neuron organization. Cambridge: Cambridge University Press.

KonORSKI, J. (1967). Integrative activity of the brain. Chicago: University of Chicago Press.

Mackintosh, N. J. (1974). The psychology of animal learning. New York: Academic Press.

Pavlov, I. P. (1927). Conditioned reflexes (G. V. Anrep, Trans.). London: Oxford University Press.

ResCORLA, R. A. (1993). Inhibitory associations between $S$ and R in extinction. Animal Learning \& Behavior, 21, 327-336.

RESCORLA, R. A. (1997). Spontaneous recovery after Pavlovian conditioning with multiple outcomes. Animal Learning \& Behavior, 25, 99-107.

Rescorla, R. A., \& Cunningham, C. L. (1978). Recovery of the US representation over time during extinction. Learning \& Motivation, 9, 373-391.

RESCORLA, R. A., \& WAgner, A. R. (1972). A theory of Pavlovian conditioning: Variations in the effectiveness of reinforcement and nonreinforcement. In A. H. Black \& W. F. Prokasy (Eds.), Classical conditioning II (pp. 64-99). New York: Appleton-Century-Crofts.

RobBins, S. J. (1990). Mechanisms underlying spontaneous recovery in autoshaping. Journal of Experimental Psychology: Animal Behavior Processes, 16, 235-249.

SPEAR, N. E. (1971). Forgetting as retrieval failure. In W. K. Honig \& P. H. R. James (Eds.), Animal memory (pp. 45-109). New York: Academic Press.

Underwood, B. J., \& Freund, J. S. (1968). Effect of temporal separation of two tasks on proactive inhibition. Journal of Experimental Psychology, 78, 50-54.

(Manuscript received February 27, 2004; revision accepted for publication June 8, 2004.) 